\title{
EFEITOS DO METILFENIDATO NO DESEMPENHO MOTOR DE CRIANÇAS COM TDAH
}

\section{Simeia Gaspar Palácio}

Departamento de Fisioterapia do Centro Universitário Cesumar (UniCesumar), Maringá (PR), Brasil.

E-mail: simeia.vieira@yahoo.com

Nayra Oliveira Ochi

Géssika Lorena Vieira

Departamento de Fisioterapia do Centro Universitário Cesumar (UniCesumar), Maringá (PR), Brasil.
RESUMO: O presente estudo teve como objetivo analisar a influência do Metilfenidato no desempenho motor de crianças com Transtorno de Déficit de Atenção e Hiperatividade. Foram selecionadas 21 crianças, de 7 a 10 anos, provenientes de três escolas municipais de Maringá (PR), de ambos os gêneros, com diagnóstico clínico confirmado de TDAH, sendo as mesmas submetidas a duas avaliações do desenvolvimento motor, utilizando o instrumento "Movement Assessment Battery for Children", em dois momentos distintos, com e sem a utilização do Metilfenidato. Os respectivos testes motores foram realizados no próprio ambiente escolar e os resultados foram bastante diversificados, sendo que na destreza manual os escores não sofreram modificação com e sem utilização do medicamento, nas habilidades de lançar e receber houve uma diminuição na pontuação do teste com o uso do fármaco, enquanto em relação ao equilíbrio observou-se $o$ inverso. Dessa forma, mediante a pequena amostra investigada, mais pesquisas são necessárias visando explorar os efeitos dose-resposta da correspondente medicação em diferentes atividades físicas ou contextos.

PALAVRAS-CHAVE: Farmacoterapia; Movimento; Transtorno do Déficit de Atenção com Hiperatividade.

\section{EFFECTS OF METHYLPHENIDATE IN THE MOTOR PERFORMANCE OF ADHD CHILDREN}

ABSTRACT: The influence of methylphenidate on the motor performance of children with Attention Deficit and Hyperactivity Disorder is analyzed. Twenty-one children, aged $7-10$ years, males and females, from three municipal schools in Maringá PR Brazil, clinically diagnosed with $\mathrm{ADHD}$, were selected. They underwent two evaluations of motor development by the Movement Assessment Battery for Children at two different instances, with and without methylphenidate. Motor tests were undertaken in school and results were highly different. Scores in manual dexterity were not different with or without drugs; throwing and receiving ability showed a lowering in test when the drug was applied, with the inverse occurring in the case of equilibrium. Since the sample was small, more research is required to explore the dose-effects of the drug during different physical activities.

KEY WORDS: Pharmacotherapy; Movement; Attention Deficit and Hyperactivity Disorder. 


\section{INTRODUÇÃO}

De acordo com Vera et al. (2006), o Transtorno de Déficit de Atenção e Hiperatividade (TDAH) é atualmente considerado como um distúrbio do desenvolvimento da infância que pode persistir na vida adulta em mais de $50 \%$ das circunstâncias. É observado em torno de 3 a $6 \%$ das crianças com idade escolar, tendo como uma característica a dificuldade em manter-se atento, a hiperatividade e a impulsividade (ROHDE et al., 2004).

Conforme Oliver (2007), tal transtorno também é denominado de Déficit de Atenção e Aprendizagem com Hiperatividade (DDAH), tendo em vista que a vida escolar dessas crianças costuma ser altamente prejudicada.

As causas do transtorno são de origem multifatorial e ainda interrogadas na literatura, estando associadas a fatores ambientais e genéticos, lesões neurológicas decorrentes de problemas na gestação ou nos estágios iniciais do desenvolvimento neonatal, além de alterações nos neurotransmissores (MELLO; MIRANDA; MUSKAT, 2005).

O TDAH raramente aparece de forma isolada estando associado a outras comorbidades como transtornos de aprendizagem, problemas motores e outras desordens neuropsiquiátricas (APA, 2000).

A ocorrência de problemas motores no TDAH é observada tanto na literatura nacional e internacional e tem sido detectada por variados instrumentos de avaliação do desenvolvimento motor e, em maior ou menor intensidade, em todos os subtipos clínicos do transtorno.

Estudos clínicos e epidemiológicos apontam que 30 a 50\% das crianças com TDAH apresentam algum comprometimento da coordenação motora (POLATAJKO; CANTIN, 2005; MAGALHÃES; MISSIUNA; WONG, 2006; HARVEY et al., 2007; FLIERS et al., 2009; STRAY et al., 2009) o que acarreta déficits na execução das atividades de vida diária (FLIERS et al., 2010), na qualidade de vida, danos emocionais e sociais (MRUG et al., 2007).

De acordo com Seidman, Valera e Makris (2006) e Shaw et al. (2007), a criança com TDAH apresenta um atraso na aquisição da maturidade cortical, sobretudo das regiões pré-frontais e vias relacionadas ao bloqueio de respostas inadequadas, atenção, memória operacional e precisão do controle motor.
Brossard-Racine et al. (2012) afirmam que os problemas motores evidenciados no TDAH podem ser em parte atribuídos aos sintomas de desatenção. Tal hipótese foi considerada ao investigarem os efeitos da utilização do Metilfenidato no desempenho motor de crianças com TDAH, verificando que $20 \%$ da amostra apresentaram melhora de desempenho com a medicação após três meses de utilização.

Atualmente, o tratamento de TDAH envolve o uso de medicamentos e terapias psicossociais (CONNERS, 2009) e, segundo Goldstein (2001), o medicamento é considerado o tratamento mais eficaz para hiperatividade.

A maioria dos indivíduos utiliza como tratamento medicamentoso o Metilfenidato, o qual recebe o nome comercial de Ritalina, com uma ação prolongada. Estes psicoestimulantes são rapidamente absorvidos com níveis máximos sanguíneos tendoefeito sobre ocomportamento, dentro de 1 a 3 horas, além de aumentar a atividade das células nervosas em muitas áreas do cérebro, provocando aumento na inibição no neocórtex ou nas áreas frontais do cérebro. Seus efeitos estimulantes ou inibidores da recaptação de norepinefrina e dopamina neutralizam as respostas indesejadas, permitindo que o paciente apresente atenção seletiva a estímulos adequados ou desejados (CONNERS, 2009).

Segundo Conners (2009) a neurotransmissão da dopamina e norepinefrina tem influência na atenção, na resposta à condução das sinapses, no grau de alerta e de atividade no comportamento exploratório. Especialistas acreditam que a dopamina seja importante para a atenção a informações ambientais interessantes ou aquelas relevantes para a sobrevivência. Presume-se que um déficit na função da dopamina leve à falta de ativação adequada (desatenção) e a uma resposta aleatória a estímulos irrelevantes (hiperatividade). Por sua vez, ainda de acordo com o referido autor, a Norepinefrina influencia na resposta aos estímulos das conduções sinápticas, incluindo sinais e ameaças à punição e não reforço. Como esses estímulos de modo geral causam inibição comportamental, o déficit no funcionamento da norepinefrina leva à resposta impulsiva, arriscada ou descontrolada à incapacidade de controlar impulsos. Já o aumento na responsividade desse sistema pode levar a excesso de inibição comportamental e ansiedade. 
Sabe-se que a medicação tem efeito benéfico sobre o comportamento e o desempenho escolar da criança, por possibilitar melhora da atenção. No entanto, poucos estudos abordam os efeitos do fármaco Metilfenidato sobre o desempenho motor da criança hiperativa. Dessa forma, tendo em vista que o tratamento medicamentoso é de forma geral uma das únicas opções terapêuticas utilizada para o tratamento do transtorno e da presença dos problemas motores em 30 a $50 \%$ desta população, o estudo em questão teve como objetivo analisar o desempenho motor de crianças com TDAH provenientes das escolas da rede municipal de ensino de Maringá (PR), com e sem o uso de Metilfenidato.

\section{METODOLOGIA}

O presente estudo, com delineamento transversal e aprovado pelo Comitê de Ética em Pesquisa sob parecer $\mathrm{n}^{\mathrm{o}} 058056 / 2014$, foi realizado em três escolas da rede municipal de ensino da cidade de Maringá (PR), com 21 alunos diagnosticados com o Transtorno de Déficit de Atenção e Hiperatividade e que já faziam o uso de Metilfenidato há pelo menos três meses consecutivos.

Foram incluídas na pesquisa crianças de 7 a 10 anos, de ambos os gêneros e independente do subtipo clínico do transtorno, cujos pais autorizaram a participação mediante a assinatura do Termo de Consentimento Livre e Esclarecido (TCLE), sendo excluídas crianças que apresentaram deficiência visual e auditiva grave, cardiopatias, deficiência mental, deformidades ortopédicas e outros distúrbios psiquiátricos e neurológicos graves.

Após a identificação das crianças com 0 transtorno e do consentimento dos pais ou responsáveis, foram agendadas as avaliações do desempenho motor, as quais ocorreram no próprio ambiente escolar em dias e horários previamente estabelecidos, ocorrendo em dois momentos distintos, sendo que em um dos dias a criança foi avaliada sob o efeito do fármaco e no outro não. Para avaliar a criança sem o efeito do Metilfenidato foi necessário contato telefônico com os pais e ou responsáveis, visando à suspensão do medicamento 48 horas antes da avaliação para que não houvesse nenhuma influência no desempenho motor da criança.

Para a realização dos testes motores foi utilizado o instrumento Movement Assessment Battery for
Children-2 (MABC-2) que tem sido amplamente utilizado para identificação de desordens motoras em crianças de 3 a 16 anOs (HENDERSON; SUGDEN; BARNETT, 2007).

O MABC-2 é dividido em três categorias de habilidades, sendo estas a destreza manual, habilidades com bola, incluindo atividades de lançar e receber e o equilíbrio estático e dinâmico. Nas tarefas da habilidade de destreza manual, quanto menor o escore bruto, melhor e mais alto são seus escores padrão. Já nas habilidades de lançar e receber e nas habilidades de equilíbrio, quanto maior o escore bruto, maior o escore padrão.

Os resultados de cada uma destas modalidades são somados, primeiramente dentro de cada conjunto de tarefas, para que seja possível obter os escores das habilidades de destrezas manuais totais, habilidades com bola total e equilíbrio total. Em seguida são somados esses três últimos valores, para que se obtenha o MABC total de cada criança.

Quanto à somatória de cada componente, uma maior pontuação indica um maior escore padrão e, consequentemente, um maior percentil, sendo o mesmo válido para a pontuação total do teste. Sendo assim, a pontuação dos componentes e a pontuação total do teste e seus respectivos escores padrão e percentis são diretamente proporcionais, o que indica que quanto mais alta a pontuação no teste MABC-2, melhor o desempenho da criança.

Escores do MABC-2 total abaixo do $5^{\circ}$ percentil são indicativos de uma significante dificuldade motora; valores entre o $5^{\circ}$ e o $15^{\circ}$ percentil indicam um risco motor; enquanto escores acima do $15^{\circ}$ percentil indicam um desenvolvimento motor normal, sem risco.

Ao término das avaliações, foram encaminhadas aos pais e aos diretores responsáveis pelas escolas informações sobre o desempenho das crianças, mediante a entrega de um relatório individual e os dados coletados foram tabulados e descritos em forma de tabelas.

Os dados foram tabulados no programa Microsoft Office Excel ${ }^{\circledR} 2007$ e posteriormente foram importados para o programa SAS (Statistical Analysis Software ${ }^{\circledR}$ ) para proceder às análises estatísticas, sendo calculadas medidas descritivas (média, desvio padrão e coeficiente de variação (CV). Para se verificar as diferenças nas medianas foi realizado o Teste de Wilcoxon para duas amostras emparelhadas, sendo estabelecido como nível de significância $5 \%$ ( $\alpha=0,05$, p-valor $<0,05)$. 


\section{RESULTADOS}

Dos 21 escolares, a maioria (15) cursava o $3^{\circ}$ e o $4^{0}$ ano do ensino fundamental, 19 eram do sexo masculino perfazendo $90,47 \%$ do total analisado, sendo a média de idade de nove anos e o desvio padrão de $\pm 1,15$ anos, conforme observado na Tabela 1 .

Tabela 1. Distribuição dos escolares investigados de acordo com o ano escolar cursado

\begin{tabular}{lc}
\hline Ano do Ensino Fundamental & $\begin{array}{c}\text { Quantidade de alunos } \\
\text { testados }\end{array}$ \\
\hline $2^{\circ}$ ano & 2 \\
$3^{\circ}$ ano & 7 \\
$4^{\circ}$ ano & 8
\end{tabular}

$5^{\circ}$ ano

Quanto às variáveis motoras, os resultados foram bastante diversificados. Dessa forma, na destreza manual não foi evidenciada a alteração do desempenho motor com e sem medicação, já nas habilidades de lançar e receber e, na pontuação total do MABC-2, houve uma piora do desempenho da amostra com o uso do fármaco, e o inverso, no que diz respeito ao equilíbrio, cujos resultados foram mais favoráveis sob a influência do Metilfenidato. No entanto, vale ressaltar que os resultados não foram estatisticamente significantes, em nenhuma das habilidades motoras analisadas (Tabela 2).

Tabela 2. Resultados do MABC-2 com e sem utilização do Metilfenidato

\begin{tabular}{|c|c|c|c|c|c|c|c|}
\hline Variável & Média & Desvio padrão & Mínimo & Máximo & CV (\%) & Mediana & $P$-valor \\
\hline $\begin{array}{l}\text { DM sob efeito do } \\
\text { Metilfenidato }\end{array}$ & 25,76 & 23,13 & 2 & 95 & 89,80 & 16 & \multirow{2}{*}{0,9357} \\
\hline $\begin{array}{l}\text { DM sem efeito do } \\
\text { Metilfenidato }\end{array}$ & 26,76 & 24,52 & 1 & 84 & 91,61 & 16 & \\
\hline LR sob efeito do Metilfenidato & 18,24 & 17,76 & 1 & 50 & 97,41 & 9 & \multirow{2}{*}{0,4659} \\
\hline LR sem efeito do Metilfenidato & 15,14 & 14,59 & 0,5 & 63 & 96,38 & 16 & \\
\hline EQ sob efeito do Metilfenidato & 21,26 & 14,95 & 0,5 & 50 & 70,30 & 25 & \multirow[b]{2}{*}{0,8145} \\
\hline $\begin{array}{l}\text { EQ sem efeito do } \\
\text { Metilfenidato }\end{array}$ & 21,50 & 17,98 & 0,5 & 63 & 83,63 & 16 & \\
\hline PT sob efeito do Metilfenidato & 16,05 & 14,54 & 1 & 50 & 90,59 & 9 & \multirow{2}{*}{0,7484} \\
\hline PT sem efeito do Metilfenidato & 14,64 & 12,84 & 0,5 & 37 & 87,70 & 16 & \\
\hline
\end{tabular}

*DM= Destreza manual; $\mathrm{LR}=$ lançar e receber; $\mathrm{EQ}=$ equilíbrio; $\mathrm{PT}=$ pontuação total. Valor estatisticamente significante $\mathrm{P}<0,05-$ teste de Wilcoxon.

\section{DISCUSSÃO}

Os resultados do presente estudo demonstraram que não houve influência do Metilfenidato no desempenho motor de escolares com TDAH.

Da mesma forma, Harvey et al. (2007), ao investigarem os efeitos do Metilfenidato na atenção e nas habilidades de beisebol em 17 meninos, entre 7,8 a 9,9 anos de idade, utilizando um grupo controle e placebo para fins de comparação, concluíram que o Metilfenidato teve um efeito significativo sobre as medidas de atenção, mas não sobre as medidas de desempenho motor, demonstrando que as crianças com TDAH podem estar em risco de atraso no desenvolvimento das habilidades motoras fundamentais, já que todas as do respectivo grupo foram significativamente diferentes de seus pares sem o transtorno.

Em contrapartida, Brossard-Racine et al. (2012), analisando o desempenho motor de 49 crianças, entre 6 e 11 anos, com o teste MABC, obtiveram uma redução dos déficits motores de $73,5 \%$ para $55,1 \%$ após três meses de uso de Metilfenidato. Tal hipótese foi considerada ao investigarem que $20 \%$ da amostra apresentaram melhora 
deste desempenho com a medicação, enquanto $80 \%$ da mesma não obtiveram os mesmos resultados, o que é indicativo de que apenas o tratamento farmacológico pode não ser o suficiente para amenizar os problemas motores detectados nestas crianças, sendo necessário que as mesmas realizem intervenções motoras complementares com profissionais especializados como fisioterapeutas e educadores físicos, visando minimizar os prejuízos escolares, emocionais e físicos, já que ambos estão associados.

Da mesma forma, Bart, Podoly e Bar-Haim (2013), avaliando o desempenho motor de 18 crianças com oito anos de idade com e sem utilização do Metilfenidato, detectaram que 16 deles apresentaram melhora no escore bruto total do teste MABC durante utilização do fármaco, indicando que a medicação pode interferir positivamente nos componentes da atenção e no planejamento motor, embora não tenha sido observada melhora significativa do equilíbrio estático. Nicolson e Fawcett apud Bart, Podoly e Bar-Haim et al. (2013) atribuem tal resultado ao fato da atenção não ser necessária para a obtenção do equilíbrio estático, uma vez que é controlada pelo sistema nervoso autônomo.

Fox, Adi-Japha e Karni (2014), ao analisarem a execução de uma sequência de movimentos de 16 adolescentes com TDAH, com e sem a utilização do Metilfenidato, verificaram que com a interrupção do fármaco ocorreu uma significativa diminuição na velocidade de desempenho dos movimentos da criança, porém não houve perda da precisão na realização dos mesmos.

Um dos resultados surpreendentes do estudo atual foi a pior performance apresentada pelas crianças nas habilidades de lançar e receber, quando estavam sob efeito da medicação, visto que esta tarefa por ter maior grau de complexidade, requer maior funcionamento do córtex pré-frontal e das funções cognitivas, sendo geralmente prejudicada nos indivíduos com o transtorno (RIGOLI et al., 2012). Este resultado contraditório ao esperado pode ser justificado em parte devido ao tamanho reduzido da amostra atual que pode ter limitado o poder estatístico dos testes para detectar estas diferenças.

Algumas divergências do estudo, com os citados anteriormente, a respeito da interferência da atenção no desempenho motor, podem ainda ser atribuídas à diversidade no tipo de amostra, na inclusão de comorbidades associadas e nos variados instrumentos de avaliação do desempenho motor utilizados nas pesquisas, os quais normalmente contêm testes com diferentes níveis de complexidade.

\section{CONCLUSÃO}

Comparando a amostra investigada com os estudos analisados, percebe-se que a medicação interfere no comportamento destas crianças, porém não é o único fator determinante no desempenho das habilidades motoras. Sendo assim, presume-se ser necessária uma intervenção fisioterapêutica associada à medicação para que se tenham resultados mais favoráveis no que diz respeito aos problemas motores frequentemente observados em indivíduos com TDAH.

Dessa forma, é primordial que estas crianças sejam estimuladas o mais precocemente possível quanto à prática de atividades físicas, a fim de que lhes sejam oferecidas as mesmas oportunidades de desenvolvimento das crianças com desenvolvimento motor típico, correspondentes à sua faixa etária.

Diante da variabilidade de resultados clínicos a respeito da interferência do Metilfenidato no desempenho motor de crianças com TDAH e da pequena amostra investigada, mais pesquisas são necessárias visando explorar os efeitos dose-resposta da correspondente medicação em diferentes atividades físicas ou contextos.

\section{REFERÊNCIAS}

APA: American Psychiatric Association. Diagnostic and statistical manual of mental disorders. 4. ed. Washington, (DC): APA, 2000.

BART, O.; PODOLY, T.; BAR-HAIM, Y. Influence of methylphenidate on motor performance and attention in children with developmental coordination disorder and attention deficit hyperactive disorder. Res. Dev. Disabil., v. 34, n. 6, p. 1922-1927, 2013.

BROSSARD-RACINE， M.; SHEVEL， M.; SNIDER， L.; 
BÉLANGER, S. A.; MAJNEMER, A. Motor skills of children newly diagnosed with Attention Deficit Hyperactivity Disorder prior to and following treatment with stimulant medication. Res. Dev. Disabil., v. 33, n. 6, p. 2080-2087, 2012.

CONNERS, C. K. Transtorno de déficit de atenção/ hiperatividade: as mais recentes estratégias de avaliação e tratamento. $3^{\mathrm{a}}$ ed. Porto Alegre: Artmed, 2009.

FLIERS, E. A.; HOOG, M. L.; FRANKE, B.; FARAONE, S. V.; ROMMELSE, N. N.; BUITELAAR, J. K.; NIJHUIS-VAN DER SANDEN, M. W. Actual Motor Performance and SelfPerceived Motor Competence in Children with healthy siblings and peers. J Dev. Behav. Pediatr., v. 31, n. 1, p. 35-40, 2010.

FARAONE, S. V.; BUITELAAR, J. K. Undertreatment of Motor Problems in Children with ADHD. Child Adolesc. Ment. Health, v. 15, n. 2, p. 85-90, 2009.

FOX, O.; ADI-JAPHA, E.; KARNI, A. The effect of a skipped dose (placebo) of methylphenidate on the learning and retention of a motor skill in adolescents with Attention Deficit Hyperactivity Disorder. Eur. NeuroPsychopharm, v. 24, n. 3, p. 391-396, 2014.

GOLDSTEIN, S. Hiperatividade: como desenvolver a capacidade de atenção da criança. $7^{\mathrm{a}}$ ed. Campinas: Papirus, 2001. 245p. (Série Educação Especial).

HARVEY, W. J.; REID, G.; GRIZENKO, M.; MBEKOU, V.; TER-STEPANIAN, M.; JOOBER, R. Fundamental movement skills and children with attention-deficit hyperactivity disorder: peer comparisons and stimulant effects. J Abnorm. Child. Psychol., v. 35, n. 5, p. 871-82, 2007.

HENDERSON, S. E.; SUGDEN, D. A.; BARNETT, A. L. Movement Assessment Battery for Children. 2nd. ed. San Antonio: Harcourt Assessment, 2007.

MAGALHÃES, L. C.; MISSIUNA, C.; WONG, S. Terminology used in research reports of developmental coordination disorder. Dev. Med. Child. Neurol., v. 48, n. 11, p. $937-$ 941, 2006.

MELLO, C. B.; MIRANDA, M. C.; MUSKAT, M. Neuropsicologia do desenvolvimento. São Paulo:
Memnon, 2005.

MRUG, S.; HOSA, B.; PELHAM, W. E.; GNAGY, E. M.; GREINER, A. R. Behavior and peer status in children with ADHD: continuity and change. J Atten. Disord., v. 10, $\mathrm{n}$. 4, p. 359-371, 2007.

OLIVER, L. Distúrbios de Aprendizagem e de Comportamento. Rio de Janeiro: WAK, 2007.

POLATAJKO, H. J.; CANTIN, N. Developmental coordination disorder (dyspraxia): an overview of the state of the art. Semin. Pediatr. Neurol., v. 12, n. 4, p. 250-258, 2005.

RIGOLI, D.; PIEK, J. P.; KANE, R.; OOSTERLAAN, J. An examination of the relationship between motor coordination and executive in adolescents. Dev. Med. Child. Neurol., v. 54, n. 11, p. 1025-1031, 2012.

ROHDE, L. A.; CONSTANTINO, E.; FILHO, M.; BENETTI, L.; GALLOIS, C.; KIELING, C. Transtorno de déficit de atenção/hiperatividade na infância e na adolescência: considerações clínicas e terapêuticas. Rev. psiquiatr. clín., v. 31, n. 3, p. 124-131, 2004.

SEIDMAN, L. J.; VALERA, E. M.; MAKRIS, N. Dorsolateral prefrontal and anterior cingulate cortex, volumetric abnormalities in adults with attention-deficit/hyperactivity disorder identified by magnetic resonance imaging. Biol. Psychiatr., v. 60, n. 10, p. 1071-1080, 2006.

SHAW, P.; MALEK, M.; WATSON, B.; SHARP, W.; EVANS, A.; GREENSTEIN, D. Attention-deficit/hyperactivity disorder is characterized by a delay in cortical maturation. Proc. Nati. Acad. Sci. USA, v. 104, n. 49, p. 19649-19654, 2007.

STRAY, L. L.; STRAY, T.; IVERSEN, S.; RUUD, A.; ELLERSTEN, B.; TONNESSEN, E. The motor function neurological assessment (MFNU) as an indicator of motor function problems in boys with ADHD. Behav. Brain. Funct., v. 5, n. 22, p. 1-13, 2009.

VERA, C. F. D.; CONDE, G. E. S.; WAJNSZTEJN, R.; NEMR, K. Transtornos de aprendizagem em presença de respiração oral em indivíduos com diagnóstico de transtornos de déficit de atenção/hiperatividade (TDAH). 
Rev. CEFAC, v. 8, n. 4, p. 441-455, 2006.

Recebido: 26 de novembro de 2015

Revisado: 11 de fevereiro de 2016

Aceito: 07 de abril de 2016 